\title{
Origem e distribuição do nervo axilar em tamanduá-bandeira (Myrmecophaga tridactyla)
}

\author{
Luciana Aparecida Rosa ${ }^{1 *}$ \\ Frederico Ozanam Carneiro e Silva ${ }^{2}$ \\ André Luiz Quagliatto Santos ${ }^{2}$ \\ Daniela Cristina de Oliveira Silva ${ }^{3}$ \\ Lázaro Antônio dos Santos ${ }^{2}$ \\ Frederico Balbino Lizardo ${ }^{3}$

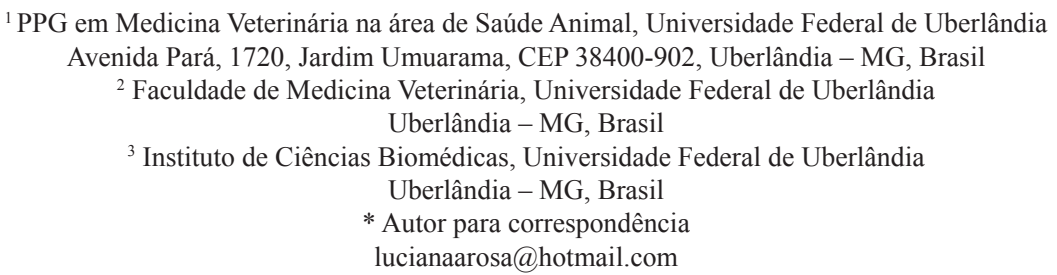

Submetido em 14/02/2012

Aceito para publicação em 19/06/2012

\section{Resumo}

O tamanduá-bandeira (Myrmecophaga tridactyla), mamífero pertencente à ordem Xenarthra, família Myrmecophagidae, está entre as espécies ameaçadas de extinção. Partindo desse pressuposto, o conhecimento da sua anatomia é de extrema insigne, principalmente do membro torácico que desempenha funções importantes na sua alimentação e como único meio de defesa. Assim, objetivou-se estudar a origem e distribuição do nervo axilar em dois tamanduás-bandeira (Myrmecophaga tridactyla), um macho e uma fêmea, pertencentes ao Laboratório de Ensino e Pesquisa de Animais Silvestres (LAPAS). Esse material consta de cadáveres fixados e conservados em solução aquosa de formol a $10 \%$ e a dissecação seguiu os procedimentos usuais em anatomia. O nervo axilar originou-se em $100 \%$ dos animais dos ramos ventrais do sexto (C6) e sétimo (C7) nervos espinhais cervicais. O referido nervo apresentou simetria em relação a sua origem e emitiu ramos para os músculos redondo maior, redondo menor e deltóide em $100 \%$ dos espécimes. Em todos os animais o nervo axilar emitiu o ramo cutâneo cranial do antebraço.

Palavras-chave: Nervos espinhais; Plexo braquial; Sistema nervoso periférico; Xenarthra

\section{Abstract}

Origin and distribution of the axillary nerve in the giant anteater (Myrmecophaga tridactyla). The giant anteater (Myrmecophaga tridactyla), a mammal belonging to the order Xenarthra and family Myrmecophagidae, is an endangered species. For this reason, additional knowledge about its anatomy is of interest, especially the forelimb, which plays important roles in feeding and defense. The goal of this study was to learn more about the 
origin and distribution of the axillary nerve of Myrmecophaga tridactyla by studying two individuals (one male and one female) that belong to the Research Laboratory of Wild Animals (UFU). The study material consisted of corpses fixed and preserved in $10 \%$ aqueous formalin solution. Dissection of the material followed standard procedures. In both animals, the axillary nerve was found in the ventral branch of the sixth cervical (C6) and seventh (C7) spinal nerve. This nerve showed symmetry in relation to its position in the two specimens and branched into the teres major, teres minor and deltoid muscles. In both specimens the axillary nerve originated in the cranial cutaneous branch of the lower leg.

Key words: Brachial plexus; Peripheral nervous system; Spinal nerves; Xenarthra

\section{Introdução}

O tamanduá-bandeira (Myrmecophaga tridactyla), mamífero pertencente à ordem Xenarthra, família Myrmecophagidae, é encontrado na América Central (Belize e Guatemala) e na América do Sul (Andes, Equador, Venezuela, Bolívia, Paraguai, Argentina, Uruguai, Colômbia e Brasil). De todas as espécies de tamanduás, o tamanduá-bandeira (Myrmecophaga tridactyla) possui o maior tamanho, medindo de 1 a $1,2 \mathrm{~m}$, mais a cauda com 65 a $90 \mathrm{~cm}$, seu peso varia de 32 e 45kg (CUBAS et al., 2006). Em sua conformação compacta, destaca-se um focinho longo, e sua pelagem é acinzentada com tonalidade mais escura no dorso, com uma faixa diagonal preta, com as bordas brancas, que se encontra no peito. Como alimentam-se basicamente de uma grande variedade de formigas e de térmitas, facilmente detectados pelo apurado olfato, utilizam suas poderosas garras anteriores para destruir parcialmente formigueiros ou cupinzeiros, retirando suas presas com sua longa e viscosa língua (ORR, 1986; HILDEBRAND, 1995; POUGH, 1999).

A destruição do hábitat, que provavelmente leva à modificação de comunidades de formigas e cupins, bem como a caça e atropelamentos frequentes em rodovias, têm ameaçado a vida desta espécie (MACHADO, 2008; IBAMA, 2012).

Neste sentido, é necessária a ampliação de informações anatômicas que possam contribuir com medidas de preservação e proteção desta espécie. Apesar de existirem alguns estudos sobre a anatomia do tamanduábandeira (Myrmecophaga tridactyla) abordando a anatomia do coração (PAREJA et al., 2008) e suprimento sanguíneo do intestino grosso (FERREIRA et al., 2010), observa-se uma escassez na literatura sobre a morfologia do sistema nervoso periférico, especialmente do nervo axilar. Esta questão parece ser importante, já que o membro torácico destes animais desempenha importantes funções como defesa e alimentação (YOUNG, 1980). Assim, o objetivo deste trabalho foi descrever a origem e distribuição dos nervos axilares em tamanduá-bandeira (Myrmecophaga tridactyla).

\section{Material e Métodos}

Para o estudo utilizou-se dois espécimes de tamanduá-bandeira (Myrmecophaga tridactyla), um macho e uma fêmea, adultos, pertencentes ao Laboratório de Estudo e Pesquisa em Animais Silvestres (LAPAS) da Universidade Federal de Uberlândia (UFU). Os espécimes, levados a óbito por atropelamentos em rodovia, foram doados pelo IBAMA-MG e encontramse fixados e conservados em solução aquosa de formol a 10\%. O estudo foi aprovado pelo Comitê de Ética de Uso em Animais da Universidade Federal de Uberlândia protocolo $n^{\circ}$ 039/11.

A dissecação foi realizada bilateralmente segundo os planos habituais de incisão, que partiram da pele, próximo ao esterno, até atingir o plexo braquial no espaço axilar dos quatro antímeros, direito e esquerdo. Após as incisões, os tecidos conjuntivo e adiposo da região axilar foram removidos, ocorrendo à individualização dos nervos que compõem o plexo braquial (GODINHO et al., 1987; SCAVONE et al., 2008).

Primeiramente, o nervo axilar foi dissecado no sentido proximal para a observação de sua origem, bem como das raízes ventrais dos nervos espinhas cervicais que contribuíram para a sua formação. Desta forma, foram evidenciadas as vértebras cervicais, o primeiro 
par de costelas, e os músculos que encobrem os forames intervertebrais (SANTANA et al., 2003; SCAVONE et al., 2008).

Posteriormente, realizou-se a dissecação do nervo axilar distalmente, observando-se sua distribuição para os músculos das faces medial e lateral do ombro. A nomenclatura adotada para a descrição dos resultados esteve de acordo com o International Committee On Veterinary Gross Anatomical Nomenclature (2005).

A análise dos dados foi realizada de forma descritiva em termos de porcentagem simples para a origem e distribuição do nervo axilar.

\section{Resultados}

Após avaliação dos dois espécimes de tamanduábandeira (Myrmecophaga tridactyla), verificou-se que todos os casos apresentaram sete vértebras cervicais (Figura 1).

Nos espécimes estudados o nervo axilar, originouse em todos os antímeros (100\%) dos ramos ventrais do sexto nervo espinhal cervical (C6) e do sétimo nervo espinal cervical (C7) (Figura 2).
FIGURA 1: Fotografia da face ventral da coluna cervical do tamanduá-bandeira (Myrmecophaga tridactyla) mostrando as sete vértebras cervicais $(\mathrm{C} 1, \mathrm{C} 2, \mathrm{C} 3$, $\mathrm{C} 4, \mathrm{C} 5, \mathrm{C} 6, \mathrm{C} 7)$.

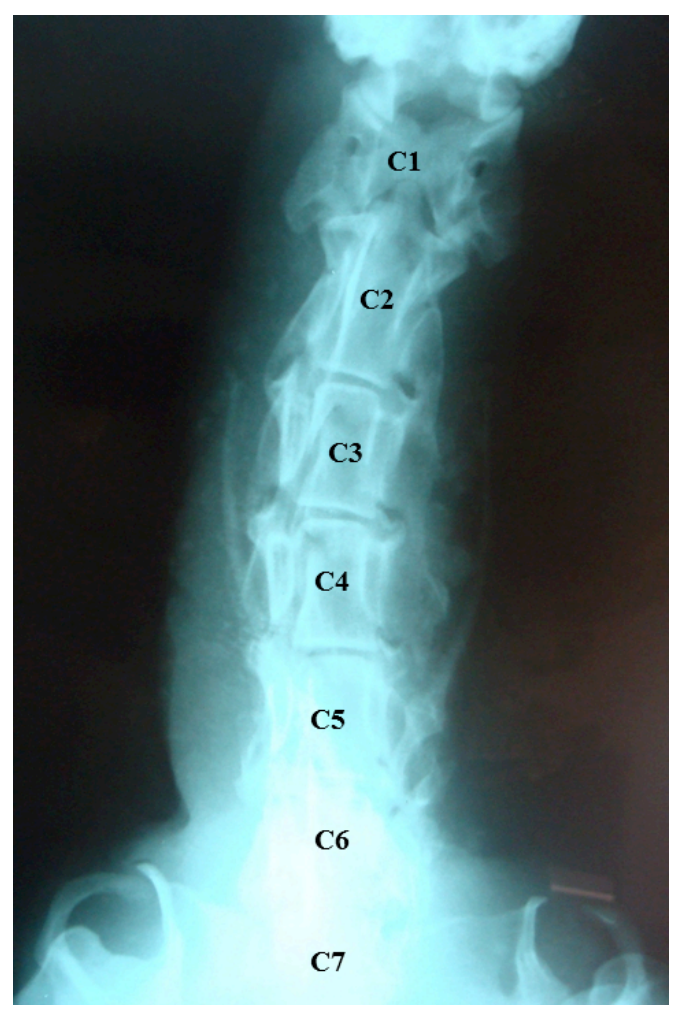

FIGURA 2: Fotografia da face ventral dos ramos ventrais dos nervos espinhais cervicais (C6 a C8) e torácicos (T1) do tamanduá-bandeira (Myrmecophaga tridactyla) demonstrando a origem do nervo axilar (axi) em C6 e C7.

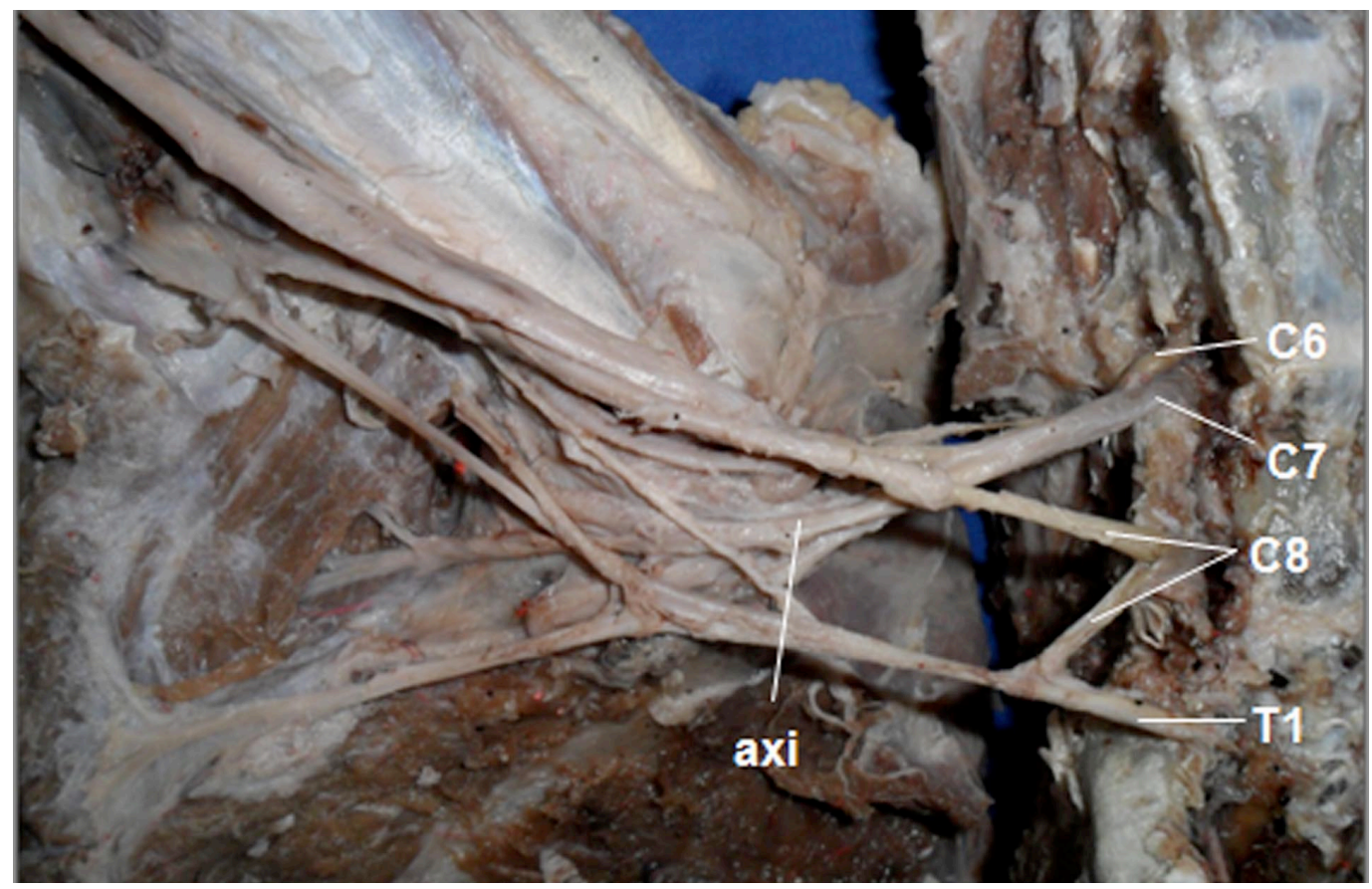


No que se refere à simetria de origem, o nervo axilar apresentou simetria em todos os espécimes estudados. Quanto à sua distribuição, foi constatado que ao longo de seu trajeto o nervo axilar cedeu ramos para os músculos redondo maior, redondo menor e deltóide, em todos os animais.

Para o músculo redondo maior o nervo axilar emitiu ramos, que variaram de quatro $(25 \%)$ a cinco $(25 \%)$ para o antímero direito e seis (50\%) para o esquerdo (Figura
3). O músculo redondo menor recebeu de um (25\%) a dois $(25 \%)$ ramos para ambos antímeros. O músculo deltóide recebeu de sete $(25 \%)$ a oito ramos $(25 \%)$ em ambos antímeros (Figura 4).

Em todos os espécimes o nervo axilar emitiu o ramo cutâneo cranial do antebraço, cuja emergência se deu entre as duas partes do músculo deltóide e distribuiu-se para a fáscia e a pele da face cranial do braço e antebraço.

FIGURA 3: Fotografia da face medial do membro torácico direito do tamanduá-bandeira (Myrmecophaga tridactyla) mostrando o nervo subescapular (sub) e nervo axilar (axi) emitindo ramos (*) para o músculo redondo maior (rma).

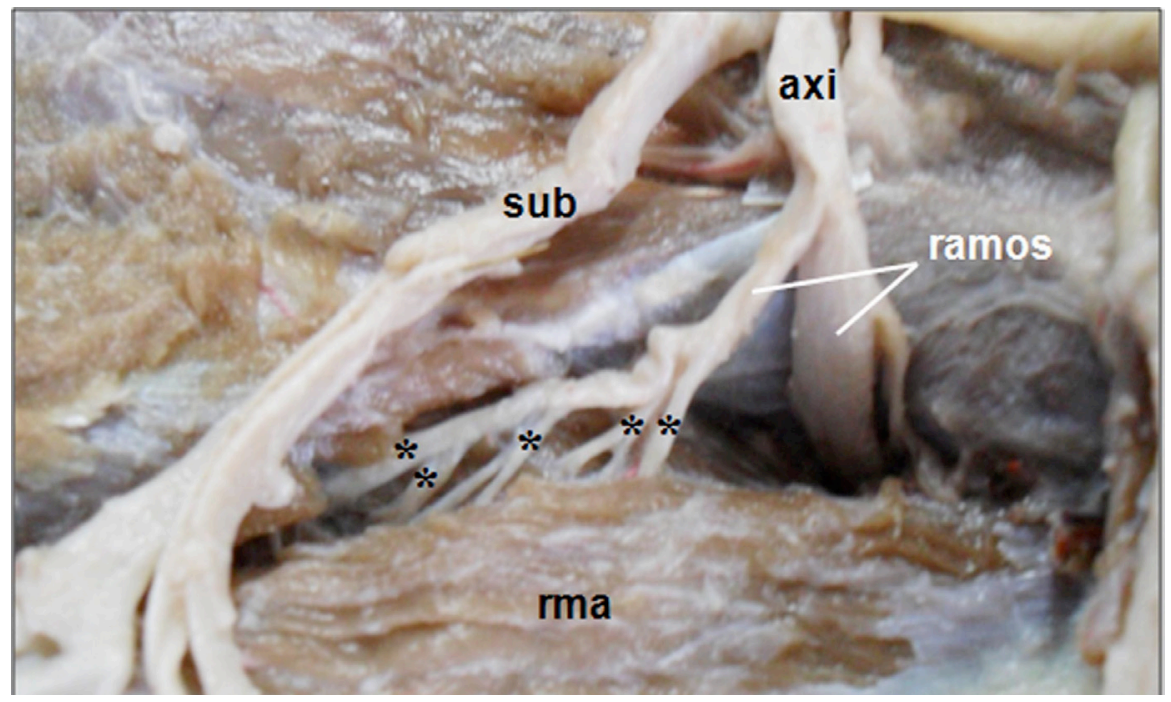

FIGURA 4: Fotografia da face lateral do membro torácico direito do tamanduá-bandeira (Myrmecophaga tridactyla) mostrando o nervo axilar (axi) emitindo um ramo* para o músculo redondo menor (rme) e se distribuindo no músculo deltóide (del).

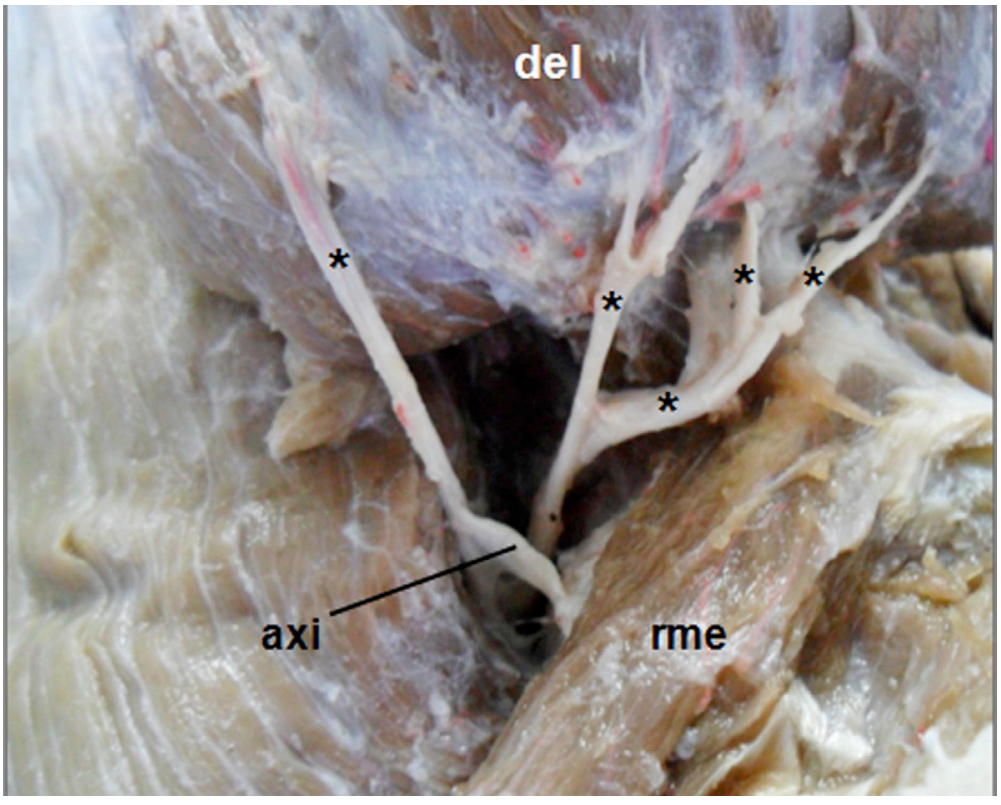




\section{Discussão}

O nervo axilar em tamanduá-bandeira (Myrmecophaga tridactyla) tem origem (C6, C7) divergente de bovinos (BRUNI; ZIMMERL, 1977; GHOSHAL, 1986; GODINHO et al., 1987; DYCE et al., 2004; KONIG et al., 2004), suínos (GHOSHAL, 1986), equinos (SCHWARZE; SCHRÖDER, 1970; BRUNI; ZIMMERL, 1977; GHOSHAL, 1986; DYCE et al., 2004), cães (SCHWARZE; SCHRÖDER, 1970; MILLER, 1979; EVANS; DELAHUNTA, 2001; DYCE et al., 2004) e cervos do pantanal Manzama sp. (OLIVEIRA et al., 2003), cuja origem do nervo axilar foi relatada a partir do sétimo (C7) e oitavo (C8) nervos espinais cervicais (Tabela 1).

Não foi observada neste estudo a origem descrita para o nervo axilar de $\mathrm{C} 6, \mathrm{C} 7$ e $\mathrm{C} 8$, em ruminantes (SCHWARZE; SCHRÖDER, 1970; BRUNI;ZIMMERL, 1977), suínos (SCHWARZE; SCHRÖDER, 1970), gatos (SCHWARZE; SCHRÖDER, 1970). Bruni e Zimmerl (1997) ainda citaram que raramente em ruminantes, o referido nervo pode originar-se de C7, C8 e T1, e segundo Lizardo (2009) em fetos de bovinos azebuados este nervo pode surgir de C6, C7, C8 e T1, sendo estas duas situações não observadas neste estudo (Tabela 1).

A origem do nervo axilar em tamanduá-bandeira (Myrmecophaga tridactyla) observada neste trabalho também difere daquele mencionado para gorila, chimpanzé e orangotango, onde o nervo axilar originase do quinto (C5) e sexto (C6) nervos espinais cervicais (HEPBURN, 1891), para ornitorrinco Ornithorhynchus anatinus, equidina Tachyglossus aculetus origina-se de C4, C5 e C6 (KOIZUMI; SAKAI, 1997), em macacoprego Cebus apella, este nervo é resultante da união de ramos de C5, C6 e C7 (RIBEIRO et al., 2005). Em capivaras, Hydrochaeris hydrochaeris (FIORETTO et al., 2003), este nervo tem origem de C5, C6 ou C6,C7 e C8 (Tabela 1).

Por outro lado, a origem do nervo axilar em tamanduá-bandeira (Myrmecophaga tridactyla) é semelhante ao encontrado em outros animais como caprinos e ovinos (GHOSHAL, 1986; GODINHO et al., 1987), catetos Tayassu tajacu (MOURA et al., 2007), chinchila Chinchilla lanigera (GAMBA et al., 2007) e paca Agouti paca (SCAVONE et al., 2008), ocorrendo participação somente do sexto (C6) e sétimo (C7) nervos espinais cervicais (Tabela 1).

As diferenças observadas na origem do nervo axilar, entre tamanduá-bandeira (Myrmecophaga tridactyla) e os dados presentes na literatura para outros animais, podem estar relacionadas a uma ascensão no sentido cranial da origem do plexo braquial devido a uma adaptação da morfologia deste plexo ao tipo de locomoção nos diferentes grupos de animais, bem como a fatores embriológicos, representado pela posição e desenvolvimento dos brotos dos membros em relação ao neuro-eixo, assim quanto mais cefálica a posição dos membros, mais craniais os nervos que participam da sua formação (CARPENTER, 1978; PARADA et al., 1989).

Em relação à simetria do nervo axilar, os dados encontrados no tamanduá-bandeira são semelhantes aos encontrados em Tayassu tajacu (MOURA et al., 2007), Agouti paca (SCAVONE et al., 2008), fetos de bovinos azebuados (LIZARDO, 2009), onde o nervo axilar apresenta simetria em todos os espécimes estudados, ou seja, as raízes nervosas que o originaram coincidiram em número em ambos os antímeros. No entanto, em

TABELA 1: Origens do nervo axilar, semelhanças e divergências em relação ao tamanduá-bandeira (Myrmecophaga tridactyla).

\begin{tabular}{ccccccc}
\hline \multicolumn{7}{c}{ Origem do nervo axilar } \\
C4, C5, C6 & C5, C6 & C5, C6, C7 & C5, C6, C7, C8 & C6, C7 & C6, C7, C8 & C6, C7, C8, T1 \\
\hline Ornitorrinco & Gorila & Macaco-prego & Capivara & Tamanduá-bandeira & Ruminante & Bovino \\
Equidina & Chipanzé & & Chinchila & Suíno \\
& Orangotango & & Cateto & Eqüino \\
& & & Paca & Cão \\
& & & Caprino & Cervo \\
\hline
\end{tabular}


Cebus apella (RIBEIRO et al., 2005) mencionaram uma assimetria deste nervo quanto à sua origem.

Segundo Machado (2005), o nervo axilar possui uma característica de nervo plurissegmentar, sendo constituído por dois ou mais nervos espinais. Tal fato foi evidenciado neste trabalho, porém, em bovinos (SISSON; GROSSMAN, 1975) citaram que este nervo origina-se, sobretudo de C8, já em Cebus apella (Ribeiro et al., 2005) relataram, uma origem direta do nervo axilar do quinto (C5) nervo espinal cervical.

Em relação à distribuição do nervo axilar, verificouse que ao longo do seu trajeto este emitiu ramos para os músculos redondo maior, redondo menor e deltóide, corroborando com os dados para equinos (SCHWARZE; SCHRÖDER, 1970; SISSON; GROSSMAN, 1975; GHOSHAL, 1986; DYCE et al., 2004), suínos (SCHWARZE; SCHRÖDER, 1970; GHOSHAL, 1986), cães (SCHWARZE; SCHRÖDER, 1970; GHOSHAL, 1986; DYCE et al., 2004) gato (SCHWARZE; SCHRÖDER, 1970), ruminantes (SCHWARZE; SCHRÖDER, 1970; BRUNI; ZIMMERL, 1977; GHOSHAL, 1986; NICKEL et al., 1986; GODINHO et al., 1987; KONIG et al., 2004), Chinchilla lanigera (GAMBA et al., 2007), javalis (SILVA et al., 2008), Agouti paca (SCAVONE et al., 2008), fetos de bovinos azebuados (LIZARDO, 2009) e para ornitorrinco Ornithorhynchus anatinus, equidina Tachyglossus aculetus, somente para o músculo deltóide e redondo menor (KOIZUMI; SAKAI, 1997).

Entretanto, nesta pesquisa não foi observada em nenhum espécime a inervação do músculo subescapular apresentada por equinos (SCHWARZE; SCHRÖDER, 1970), cães (SCHWARZE; SCHRÖDER, 1970; MILLER, 1979; GHOSHAL, 1986; EVANS; DELAHUNTA, 2001), suínos (SCHWARZE; SCHRÖDER, 1970; GHOSHAL, 1986), gato (SCHWARZE; SCHRÖDER, 1970), ruminantes (SCHWARZE; SCHRÖDER, 1970; GHOSHAL, 1986; NICKEL et al., 1986; GODINHO et al., 1987), Chinchilla lanigera (GAMBA et al., 2007), javalis (SANTOS et al., 2008), Agouti paca (SCAVONE et al., 2008) e fetos de bovinos azebuados (LIZARDO, 2009).

Em tamanduá-bandeira (Myrmecophaga tridactyla), o nervo axilar não supre a parte cleidobraquial do músculo braquiocefálico, diferindo de equinos (SCHWARZE; SCHRÖDER, 1970; SISSON; GROSSMAN, 1975), suínos (SCHWARZE; SCHRÖDER, 1970; GHOSHAL, 1986), cães (SCHWARZE; SCHRÖDER, 1970; GHOSHAL, 1986; NICKEL et al., 1986; GODINHO et al., 1987; KONIG et al., 2004), Chinchilla lanigera (GAMBA et al., 2007), javalis (SANTOS et al., 2008), Agouti paca (SCAVONE et al., 2008), e fetos de bovinos azebuados (LIZARDO, 2009).

O tamanduá-bandeira Myrmecophaga tridactyla não apresenta a inervação dos músculos braquial (BRUNI; ZIMMERL, 1977), cabeça medial do músculo tríceps braquial em Hydrochaeris hydrochaeris (FIORETTO et al., 2003), periósteo do osso úmero em javalis (SILVA et al., 2008).

O nervo axilar nos quatro espécimes de tamanduábandeira (Myrmecophaga tridactyla) emitiu o ramo cutâneo cranial do antebraço, semelhante em equinos (SCHWARZE; SCHRÖDER，1970; SISSON; GROSSMAN, 1975; DYCE et al., 2004), suínos (GHOSHAL, 1986), bovinos (GHOSHAL, 1986), cães (MILLER, 1979; GHOSHAL, 1986; EVANS; DELAHUNTA, 2001; DYCE et al., 2004), fetos de bovinos azebuados (LIZARDO, 2009).

Os dados obtidos, quanto à origem e distribuição do nervo axilar, são importantes em casos de afecções, abordagens clínicas ou cirúrgicas que envolvam ou comprometam a estrutura acima citada.

Assim, concluiu-se que os nervos axilares do tamanduá-bandeira (Myrmecophaga tridactyla) originaram-se dos ramos ventrais de $\mathrm{C} 6$ e $\mathrm{C} 7$, e distribuíram-se nos músculos redondo maior, redondo menor, deltóide e pele da face cranial do braço e antebraço pelo ramo cutâneo cranial do antebraço.

\section{Referências}

BRUNI, A. C.; ZIMMERL, U. Anatomia degli animali domestici. 2. ed. v. 2. Milano: Casa Editrice Dottor Francesco Vallardi , 1977. $595 \mathrm{p}$.

CARPENTER, M. B. Neuroanatomia humana. 7. ed. Rio de Janeiro: Interamericana, 1978. $770 \mathrm{p}$.

CUBAS, Z. S.; SILVA, J. C. R.; CATÃO-DIAS, J. L. Tratado de animais selvagens: Medicina Veterinária. São Paulo: Roca, 2006. $1376 \mathrm{p}$. 
DYCE, K. M.; SACK, W. O.; WENSING, C. J. G. Tratado de anatomia veterinária. 3. ed. Rio de Janeiro: Elsevier, 2004. 813 p. EVANS H. E.; DELAHUNTA A. Guia para dissecção do cão. 5 ed. Rio de Janeiro: Guanabara Koogan, 2001. 206 p.

FERREIRA A. J.; SOUZA A. L. R.; RESENDE L. C.; MORTOSA A. R. Modelo de suprimento sanguíneo do intestino grosso do tamanduá bandeira (Myrmecophaga tridactyla). Ciência Rural, Santa Maria, v. 40, n. 3, p. 541-547, 2010.

FIORETTO, E. T.; SOUZA CASTRO, M. F.; GUIDO, W. L.; MAINARDI, R.; SOUZA, R. R.; RIBEIRO, A. A. C. M. Gross anatomic organization of the capybara's (Hydrochaeris hidrochaeris) brachial plexus. Anatomia, Histologia, Embryologia, Berlin, v. 32, n. 3, p.169-174, 2003.

GAMBA, C. O.; CASTRO, T. F.; RICKES, E. M.; PEREIRA, M. A. M. Sistematização dos territórios nervosos do plexo braquial em chinchila (Chinchilla lanigera). Brazilian Journal of Veterinary Research and Animal Science, São Paulo, v. 44, n. 4, p. 283-289, 2007.

GHOSHAL, N. G. Nervos espinhais. In: GETTY, R. (Ed.). Sisson/ Grossman anatomia dos animais domésticos. 5 ed. Vol. 1. Rio de Janeiro: Guanabara Koogan, 1986. 1134 p.

GODINHO, H. P.; CARDOSO, F. M.; NASCIMENTO, J. F. Anatomia dos ruminantes domésticos. Belo Horizonte: Universidade Federal de Minas Gerais, 1987. 416 p.

HEPBURN, D. The comparative anatomy of the muscles and nerves of the superior and inferior extremities of the anthropoid apes. Part I. Journal of Anatomy and Pshysology, London, p. 149-185, 1891.

HILDEBRAND, M. Análise da estrutura dos vertebrados. 3 ed. São Paulo: Atheneu, 1995. 506 p.

IBAMA. Espécies ameaçadas. 2012. Disponível em: < http://www. ibama.gov.br>. Acesso em: 4 mar. 2012.

INTERNATIONAL COMMITTEE ON THE VETERINARY GROSS ANATOMICAL NOMENCLATURE. Nomina Anatomica Veterinaria. 5. ed. Columbia: Editorial Committee Hannover, 2005. 166 p.

KOIZUMI, M.; SAKAI, T. On the morphology of the brachial plexus of the platypus (Ornithorhynchus anatinus) and the echidna (Tachyglossus aculetus). Journal of Anatomy, Maiden, v. 190, n. 3, p. 447-445, 1997.

KONIG, H. E.; LIEBICH, H. G.; CERVENY, C. Anatomia dos animais domésticos: texto e atlas colorido, órgãos e sistemas. Vol 2. Porto Alegre: Artmed, 2004. 400 p.

LIZARDO, F. B. Origem e distribuição dos nervos axilares em fetos de bovinos azebuados. 2009. 43 f. Dissertação (Mestrado em Saúde Animal) - Universidade Federal de Uberlândia, Brasil. 2009.

MACHADO, A. B. M. Neuroanatomia funcional. 2. ed São Paulo: Atheneu, 2005. 363 p.

MACHADO, A. B. M.; DRUMMOND, G. M.; PAGLIA, A. P. Livro vermelho da fauna brasileira ameaçada de extinção. Vol. 2. Brasília: Biodiversitas, 2008. 1420 p.

MILLER, M. E. Miller's anatomy of the dog. 2. ed. Philadelphia: W. B. Sauders, 1979. 1181 p.
MOURA, C. E. B.; ALBUQUERQUE, J. F. G.; MAGALHÃES, M. S.; SILVA, N. B.; OLIVEIRA, M. F.; PAPA, P. C. Análise comparativa da origem do plexo braquial de catetos (Tayassu tajacu). Pesquisa Veterinária Brasileira, Rio de Janeiro, v. 27, n. 9, p. 357-362, 2007.

NICKEL, R.; SCHUMMER, A.; SEIFERLE, E.; FREWEIN, J.; WILKENS, H.; WILLE, K. H. The locomotor system of the domestic mammals. Berlim: Paul Parey, 1986. 499 p.

OLIVEIRA, M. F.; MOURA, C. E. B.; MIGLINO, M. A.; ASSIS NETO, A. C.; COSTA, W. P.; BENEDICTO, H.; PEREIRA, F. T. V.; AMBRÓSIO C. E. 2003. Anatomia do plexo braquial de cervos do pantanal (Manzana sp.). International Journal of Morphology, Chile, v. 21, n. 1, p. 55, 2003.

ORR, R. T. Biologia dos vertebrados. 5. ed. São Paulo: Rocca, 1986. $518 \mathrm{p}$.

PARADA, H.; PINEDA, U. H.; LAGUNAS, E. M.; VIDAL, H. A. Variaciones anatômicas de las ramas raquídeas que constituyen los troncos de origen del plexo braquial. Anales de Anatomia Normal, Santiago, v. 7, p. 32-35, 1989.

PAREJA, C. N.; ARENÁZIO, F.; CRUVINEL, C. A. T.; OLIVEIRA, J. C.; CÂNDIDO, R. F.; MELO, A. P. F.; CENTENO, A. L. Aspectos anatômicos do coração do tamanduá-bandeira (Myrmecophaga tridactyla, Linaeus, 1758). In: CONGRESSO BRASILEIRO DE MEDICINA VETERINÁRIA, 35, 2008, Gramado. Anais... Gramado: Conbravet, 2008. Versão eletrônica.

POUGH, F. H. A vida dos vertebrados. 2. ed. São Paulo: Atheneu, 1999. $798 \mathrm{p}$.

RIBEIRO, A. R.; PRADA, I. L. S.; SILVA, Z.; BARROS, R. A. C.; SILVA, D. C. O. Origem do plexo braquial do macaco Cebus apella. Brazilian Journal of Veterinary Research and Animal Science, São Paulo, v. 42, n. 2, p. 143-149, 2005.

SANTANA, J. J.; ALBUQUERQUE, J. F. G.; MOURA, C. E. B.; COSTA, W. P.; OLIVEIRA, M. F.; BARRETO JÚNIOR, R. A.; MIGLINO, M. A. Origem do plexo braquial de mocós (Kerodon rupestres wied, 1820). Brazilian Journal of Veterinary Research and Animal Science, São Paulo, v. 40, n. 6, p. 391-396, 2003.

SCAVONE, A. R. F.; MACHADO, M. R. F.; GUIMARÃES, G. C.: OLIVEIRA, F. S.; GERBASI, S. H. B. Análise da origem e distribuição dos nervos periféricos do plexo braquial da paca (Agouti paca, Linnaeus, 1766). Ciência Animal Brasileira, Goiânia, v. 9, n. 4, p. 1046-1055, 2008.

SCHWARZE, E.; SCHRÖDER, L. Compendio de anatomia veterinária: aparato locomotor. v. 1. Zaragoza: Acribria, 1970. 318 p.

SILVA, F. O. C.; FERREIRA, F. S.; IGLESIAS, L. P.; BRITO, T. R. Distribuição do nervo axilar em javalis (Sus sus scrofa). In: CONGRESSO BRASILEIRO DE MEDICINA VETERINÁRIA, 35, 2008, Gramado. Anais... Gramado: CONBRAVET, 2008. p. $1-4$.

SISSON, S.; GROSSMAN, J. D. Anatomia de los animales domésticos. 4. ed. Barcelona: Salvat, 1975. 958 p.

YOUNG, J. Z. La vida de los vertebrados. 2. ed. Barcelona: Omega, 1980. 718 p. 